\title{
Kulturelles Kapital und Musikrezeption
}

\author{
Eine empirische Überprüfung von Bourdieus Theorie der \\ Kunstwahrnehmung ${ }^{1}$
}

\section{Von Jörg Rössel}

Zusammenfassung: In Bourdieus soziokultureller Klassentheorie wird die Reproduktion von Klassenstrukturen vor allem über Prozesse der intergenerationalen Weitergabe von kulturellem Kapital und ihrer Manifestation in Lebensstilen erklärt. Dabei wurde gegen Bourdieu behauptet, dass Lebensstile und insbesondere die Rezeption von Kunst, der von Bourdieu eine zentrale Bedeutung zugesprochen wird, kaum von der Ausstattung einer Person mit kulturellem Kapital abhängig ist. In diesem Aufsatz wird die Relevanz von kulturellem Kapital für die Kunstrezeption am Beispiel der Wahrnehmung von Opernmusik geprüft. Im Gegensatz zu den meisten vorhergehenden Studien wird daher nicht analysiert, welche Musikrichtungen Personen hören, sondern es wird untersucht, wie Musik rezipiert wird. Sowohl die Ausstattung der Akteure mit kulturellem Kapital, wie auch ihre Musikrezeption konnten in einer Publikumsumfrage unter Opernbesuchern differenziert erhoben werden. In der empirischen Untersuchung wird deutlich, dass es deutlich mehr Formen der Musikrezeption gibt, als dies in Bourdieus Kunstsoziologie mit ihrem Fokus auf eine decodierende und analysierende Rezeption behauptet wird. Die Stärke der analysierenden Rezeptionsweise kann allerdings durch die Ausstattung der Akteure mit kulturellem Kapital relativ gut erklärt werden.

\section{Einleitung}

Seit der Publikation von Bourdieus „Feinen Unterschieden“ wird in der Sozialstrukturanalyse den Lebensstilen und dem kulturellen Kapital von Personen eine besondere Bedeutung bei der Reproduktion von Klassenstrukturen zugesprochen (Bourdieu 1982). Das kulturelle Kapital einer Person besteht in der Fähigkeit, kulturelle Symbole mit einem spezifischen sozialen Status in einer bestimmten Referenzgruppe zu verstehen und selbst hervorzubringen (Lareau / Lamont 1988). Damit ist die Fähigkeit von Personen verbunden sich im Bildungssystem und auf dem Arbeitsmarkt in guten Positionen zu platzieren (Bourdieu / Passeron 1977, 1979). Allerdings ist Bourdieus These, dass kulturelles Kapital und die auf dieser Grundlage konstruierten Lebensstile zentral für die Reproduktion von Klassenstrukturen sind, scharf kritisiert worden. So wurde darauf verwiesen, dass die Hochkultur, die laut Bourdieu im Frankreich der sechziger Jahre von großer Bedeutung für die Klassenreproduktion war, in den Vereinigten Staaten nur eine untergeordnete Rolle einnimmt, da in dieser Gesellschaft selbst die Eliten eher die Populär- und Massenkultur rezipieren (Erickson 1996; Lamont 1992; Peterson / Kern 1996). Halle (1993) hat sogar die These vertreten, dass klassenspezifische Unterschiede in der Rezeption von Kunst marginal seien, so dass auf dieser Grundlage auch keine klassenspezifische Distinktion möglich wäre. Gegen diese Kritik an Bourdieus Theorie der kulturellen Reproduktion hat Holt $(1997,1998)$ zwei zentrale Argumente ins Feld geführt: Erstens basiert der Prozess der Reproduktion von Klassen in unterschiedlichen Gesellschaften und historischen Epochen nicht immer auf den gleichen kulturellen Gehalten. Die große Bedeutung, die klassischer Musik und bildender Kunst in den „Feinen Unterschieden“ zugesprochen wird, dürfte lediglich ein Merkmal der französischen Gesellschaft der sechziger Jahre sein. Darüber hinaus kommt es zweitens aber nicht allein darauf an, was die sozialen Akteure konsumieren und rezipieren, sondern wie diese etwas konsumieren und rezipieren. Diese Unterschiede im

1 Für die Überlassung der Daten möchte ich mich bei Hanif Shangama bedanken. Wertvolle Hinweise und Anregungen habe ich von Gunnar Otte, dem Gutachter und den Herausgebern der Sozialen Welt erhalten. 
Geschmack und in der Form der Rezeption konnte Holt auf der Grundlage qualitativer Interviews in einem Vergleich von Personen mit hohem und Personen mit einem niedrigen kulturellen Kapital auch an Beispielen aus der Populärkultur und dem Massenkonsum verdeutlichen.

Folgt man der Diskussion über die Theorie von Pierre Bourdieu, so hängt die Rolle der Kultur im Prozess der Klassenreproduktion ganz offensichtlich von den klassenspezifischen Unterschieden im Geschmack und der Rezeption von Kunst ab. Daher soll in diesem Aufsatz mit Bourdieus Theorie der Kunstrezeption das zentrale, umkämpfte Theoriestück dieser Diskussion aufgegriffen werden. Im Anschluss an eine kurze Skizze seiner Theorie der kulturellen Klassenreproduktion (2.1.) und der kritischen Diskussion darüber (2.2.) werden die zentralen Gehalte von Bourdieus Theorie der Kunstrezeption dargestellt (2.3.). Die auf dieser Grundlage entwickelten Hypothesen sollen dann am Beispiel der Rezeption von Opern systematisch geprüft werden. Opernmusik ist ein ausgesprochen gutes Beispiel, um Bourdieus Thesen über die Rolle von kulturellem Kapital im Prozess der Kunstrezeption zu testen, da er der Musik eine besonders klassifizierende Wirkung zuspricht (Bourdieu 1982). Dabei kann in dieser empirischen Studie auf eine Itembatterie aus der Musikpsychologie zurückgegriffen werden, die eine besonders differenzierte Erfassung der Hörweisen von Opernbesuchern erlaubt. In der Zusammenfassung (5.) soll nicht nur die Relevanz der empirischen Ergebnisse für die Theorie der Kunstrezeption von Bourdieu diskutiert werden, sondern auch theoretische Perspektiven, die von seiner Theorie nicht erfasste Aspekte der Musikrezeption erfassen können.

\section{Klassenstruktur, kulturelles Kapital und Kunstrezeption}

\subsection{Kulturelles Kapital und die Reproduktion der Klassenstruktur}

Im Mittelpunkt der Sozialtheorie von Pierre Bourdieu stehen die Verhältnisse und Abhängigkeiten von Klassenpositionen, Lebensstilen, Bildung und Kulturpartizipation. Bourdieu hat sich in seinem Werk schon früh mit der stabilen Reproduktion der Klassenstrukturen moderner Gesellschaften über den Mechanismus der Ungleichheit der Bildungschancen beschäftigt (Bourdieu / Passeron 1977, 1979). Im Anschluss an Marx konzipiert er die Klassenstruktur über die Verteilung von verschiedenen Kapitalarten (Bourdieu 1983). Dabei unterscheidet er vor allem ökonomisches, soziales und kulturelles Kapital (Fröhlich 1994: 34ff; Bourdieu 1983). Obwohl Bourdieu den klassischen Kapitalbegriff erweitert, bleibt in seiner Konzeption das ökonomische Kapital in Form von Geld und Eigentumsrechten dominant. Mit sozialem Kapital bezeichnet er die formalen und informalen Beziehungsnetze von Personen, die für den Erwerb anderer Kapitalsorten von Relevanz sein können. Schließlich differenziert er das kulturelle Kapital in drei Formen aus: erstens das institutionalisierte Kulturkapital in Form von Bildungstiteln, zweitens das objektivierte Kulturkapital in Form von Gegenständen (Bildern, Büchern, Musikinstrumenten, Partituren) und drittens das inkorporierte Kulturkapital. Letzteres meint einverleibte Fähigkeiten, Dispositionen und Fertigkeiten, welche eine Person z.B. zur Rezeption von Kunstwerken befähigen; dabei bezieht sich Bourdieu vor allem auf Werke der klassischen Hochkultur, die er als legitime Kultur bezeichnet (Bourdieu 1982: 36, 80).

Die Klassenstruktur von Gesellschaften ergibt sich in Bourdieus Konzeption aus der Verteilung der Kapitalsorten. Während er die drei von ihm postulierten Klassenformationen (herrschende Klasse, Mittelklassen, Arbeiter und Bauern) entlang des Umfangs ihres ökonomischen und kulturellen Kapitals vertikal ordnet, eröffnet er zusätzlich eine horizontale Dimension der Klassenstruktur, die die Verteilung dieser beiden Kapitalarten abbildet. Er sieht die verschiedenen Klassen und Klassenfraktionen der kapitalistischen Gesellschaft nicht allein in einem ökonomischen Klassenkampf, sondern auch in einem symbolischen Klassenkampf um die Geltung von Werten und Kultur verstrickt. Insofern kommt in den verschiedenen kulturellen Lebensstilen die Klassenstruktur der Gesellschaft zum Ausdruck. So kann man in Bourdieus 
Analyse des Raums der Lebensstile feststellen, dass in der vertikalen Perspektive die Orientierung an der traditionellen Hochkultur von unten nach oben zunimmt, doch zeigen sich dabei ausgesprochen klare horizontale Differenzierungen entsprechend der Zusammensetzung der Kapitalsorten (vgl. Bourdieu 1982: 212 f).

Die Reproduktion dieser Klassen verläuft grundsätzlich nach dem Muster der Weitergabe der Kapitalarten in ihrer Zusammensetzung und Höhe durch die Eltern an die Kinder. So erfolgt die Reproduktion der sozialen Position bei den Klassenfraktionen mit einem Übergewicht von kulturellem Kapital auf der Basis der Weitergabe dieses Kapitals, neben dem frühzeitigen Erwerb von kultureller Kompetenz vorrangig durch Investitionen in den Bildungsmarkt und den Erwerb von möglichst hohen oder höheren Bildungstiteln. Dies trifft zum Beispiel auf Intellektuelle, Künstler und Freiberufler zu, deren soziale Position durch ihre Nachkommen nur reproduziert werden kann, wenn diese vergleichbar hohe Bildungstitel erwerben (Bourdieu 1982: 442ff). Die alleinige Weitergabe des Kapitals kann aber die sozialen Positionen nicht absichern. Die Individuen der jüngeren Generationen müssen entsprechend ihrer Position Strategien der Kapitalakkumulation entwickeln, die ihnen materielle oder symbolische Gewinne versprechen. Ist dies nicht möglich, sind die Individuen gezwungen, auf eine andere, gewinnträchtigere Kapitalsorte und andere soziale Felder umzustellen (Bourdieu 1982: 227ff).

In den bisherigen Ausführungen ist der Zusammenhang zwischen inkorporiertem kulturellem Kapital und dem Bildungserfolg angedeutet worden. Der in frühen Sozialisationsphasen im Elternhaus erworbene Habitus ist laut Bourdieu grundlegend für den Lernerfolg in der Schule und den Erwerb von Bildungstiteln. Schüler und Studenten mit hohem inkorporiertem kulturellem Kapital sind in ihren Interessen vielseitiger und verfügen über die Fähigkeit, Werke der klassischen Hochkultur zu verstehen und zu genießen (Bourdieu / Passeron 1977). Genau diese Fähigkeiten kommen aber letztlich in der Kommunikation zwischen Professoren und Studenten und in der Struktur der Examen zur Geltung, so dass sich das inkorporierte kulturelle Kapital in messbaren Bildungserfolgen niederschlägt. Insofern kann dann schließlich in Bourdieus theoretischer Konzeption die soziale Ungleichheit der Bildungschancen vor allem durch den Unterschied des im Elternhaushalt erworbenen kulturellen Kapitals erklärt werden.

\subsection{Die kritische Diskussion der Theorie der kulturellen Reproduktion}

Die Bedeutung des kulturellen Kapitals für den Bildungserfolg und damit ein zentrales Element der Theorie der kulturellen Reproduktion wurde mittlerweile in einer Reihe von Studien in mehreren Ländern mit meist positiven Resultaten empirisch überprüft (Hartmann 1999: 100 f; Lareau / Weininger 2003; dagegen aber Katsilis / Rubinson 1990; Robinson / Garnier 1985; Dumais 2005; Parcel / Dufur 2001). Dabei wurde das kulturelle Kapital im Hinblick auf den Bildungserfolg zumeist als hochkulturelles Kapital spezifiziert (Gebesmair 2004). Umstritten ist in der Forschung die Frage, welcher Aspekt des hochkulturellen Kapitals relevant für den Bildungserfolg ist: So hat DiMaggio vor allem auf die Bedeutung der Partizipation an hochkulturellen Aktivitäten verwiesen (DiMaggio 1982 a; DiMaggio / Mohr 1985; DiMaggio / Mukhtar 2004; Rössel / Beckert-Zieglschmid 2002) während Sullivan (2001) und de Graaf / de Graaf / Kraaykamp (2000) insbesondere die Bedeutung des Lesens betont haben. Insgesamt muss allerdings resümiert werden, dass das kulturelle Kapital in diesen Studien immer nur eine begrenzte Erklärungskraft im Hinblick auf die jeweils abhängige Variable hat, daneben sind auch andere Faktoren, wie die intellektuellen Fähigkeiten, die Klassenposition des Elternhaus und die Bildung der Eltern von Bedeutung. Die bisherigen empirischen Studien sprechen also nur für eine eingeschränkte Bedeutung der Theorie der kulturellen Reproduktion.

Neben diesen empirischen Einschränkungen für die Gültigkeit der Theorie der kulturellen Reproduktion sind noch grundsätzlichere Kritikpunkte an Bourdieus Konzeption formuliert worden. So haben verschiedene Autoren darauf verwiesen, dass die exklusive Orientierung an 
der Hochkultur in den Vereinigten Staaten auch für Mitglieder der oberen Klassen eher selten ist und daher diese Formen der Kultur für die Klassenreproduktion nur von eingeschränkter Bedeutung sein könnten (Gartman 1991; Ostrower 1998; Peterson / Kern 1996; Erickson 1996; Lamont 1992; Halle 1984: 48ff). Dagegen spielen z.B. in den Vereinigten Staaten selbst in den Bildungsschichten Formen des Massenkonsums und der Populärkultur eine sehr viel größere Rolle als im Frankreich der sechziger Jahre, das den Gegenstand der „Feinen Unterschiede“ von Pierre Bourdieu darstellt. Vergleichbare Argumente finden sich auch für europäische Gesellschaften (Schulze 1992). Diese kritischen Auseinandersetzungen mit Bourdieus Theorie der kulturellen Reproduktion relativiert allerdings Douglas Holt anhand zweier zentraler Gegenargumente: Erstens handelt es sich beim hochkulturellen Kapital nur um ein besonderes feldspezifisches Kapital, das keineswegs alle Erscheinungsformen kulturellen Kapitals abdeckt (Holt 1997: 97, 100). Holt betont, dass neben der Hochkultur auch andere Formen der Kultur eine Funktion als kulturelles Kapital ausüben können. Zweitens weist er nachdrücklich darauf hin, dass das kulturelle Kapital nicht allein Grundlage für den Konsum und die Rezeption unterschiedlicher Formen von kulturellen Produkten ist, sondern vor allem die Grundlage für grundlegend verschiedene Formen der Rezeption (Holt 1997, 1998). Es geht also nicht allein um die Frage, was eine Person rezipiert oder konsumiert, sondern wie eine Person bestimmte kulturelle Objekte rezipiert. Damit würde sich die Kritik an Bourdieus Theorie der kulturellen Reproduktion vor allem als eine Problematisierung der Operationalisierung seiner Theorie darstellen, die erstens auf Geschmackspräferenzen und zweitens auf die Hochkultur fokussiert war (Holt 1997). Allerdings hat David Halle (1993) in einer empirischen Studie eine weitergehende Kritik an der Theorie der kulturellen Reproduktion geübt, die auch Holts Argumente in Zweifel zieht. In seiner Untersuchung über Bilder und Photographien in Wohnhäusern unterschiedlicher sozialer Klassen versucht er zu demonstrieren, dass sich selbst die Rezeptionsformen abstrakter Malerei zwischen den Klassen nicht grundlegend unterscheiden. Zwar besitzen Haushalte der Arbeiterklasse deutlich seltener abstrakte Kunstwerke als Haushalte der oberen Klassen, doch zeigen sich in den Interpretationen von abstrakter Kunst kaum Unterschiede zwischen den verschiedenen Typen von Haushalten (Halle 1993: 123, 129). Die Unterschiede in der Rezeption von abstrakter Kunst zwischen Haushalten der Arbeiterklasse einerseits und der Mittel- und Oberklasse andererseits sind so gering, dass kaum von einem durch Bildungseinrichtungen und Familien erzeugten kulturellen Kapital gesprochen werden kann, das überhaupt erst den Geschmack und die Fähigkeit zur Rezeption moderner Kunst erzeugt (Halle 1993: 137). Halle zieht also offensichtlich die der Theorie der kulturellen Reproduktion zugrunde liegende Theorie der Kunstrezeption von Pierre Bourdieu selbst in Zweifel.

Diese Ausführungen zeigen, dass die Theorie der kulturellen Reproduktion von Pierre Bourdieu scharfer Kritik ausgesetzt ist. Dabei wurde deutlich, dass ein zentrales Problem in dieser Debatte ist, ob es überhaupt klassenspezifische Unterschiede in der Rezeption von Kunstwerken gibt, die auf einer unterschiedlichen Ausstattung mit kulturellem Kapital beruhen. Insofern steht offensichtlich Bourdieus Theorie der Kunstrezeption im Mittelpunkt der Diskussion, so dass diese nun kurz skizziert werden soll.

\subsection{Pierre Bourdieus soziologische Theorie der Kunstrezeption}

Die Rolle des kulturellen Kapitals bei der Rezeption von Kunstwerken sowie dessen typische Quellen lassen sich anhand von Bourdieus Theorie der Kunstrezeption analysieren. Damit können auch präzisere Fragestellungen für die folgende empirische Studie über die Rezeption von Opernmusik entwickelt werden. Die Grundannahme von Bourdieus Theorie der Kunstrezeption ist, dass die Betrachtung und Wahrnehmung eines kulturellen Werkes immer einen Prozess der Decodierung enthält (Bourdieu 1970: 159). Damit ist aber - wie auch von Douglas Holt betont wird - prinzipiell die Möglichkeit gegeben, dass Kunstwerke in Abhängigkeit von 
der jeweiligen ästhetischen Kompetenz bzw. vom kulturellen Kapital des Betrachters unterschiedlich rezipiert und wahrgenommen werden können (Bourdieu 1970: 165). Dies gilt nicht nur für Werke der Hochkultur, sondern genauso für Produktionen im Bereich der Populärkultur (Holt 1998).

Für viele Personen der unteren und mittleren sozialen Klassen stellen Werke der zeitgenössischen Kunst aber insofern eine Quelle von Verwirrung und Missverständnis dar, als sie zumeist ihr alltägliches Wissen nutzen, um diese Kunstwerke zu entschlüsseln (Bourdieu 1970: $161 \mathrm{f}$ ). Dieses alltägliche kulturelle Kapital erlaubt allerdings nur eine Entschlüsselung der oberflächlichen Bedeutung von Kunstwerken. Dies führt typischerweise dazu, dass Personen aus den unteren und mittleren sozialen Klassen Kunstwerke vor allem dann als schön empfinden, wenn sie Dinge darstellen, die selbst als schön betrachtet werden können, wie eine attraktive Frau oder ein Sonnenuntergang (Bourdieu 1982: 53ff; Bourdieu et al. 1981). Die formalen und stilistischen Aspekte eines Kunstwerks, die aus der Stellung seines Produzenten in der Geschichte des künstlerischen Feldes erwachsen und die erst ein tiefer gehendes Verständnis und damit den Genuss des Kunstwerks ermöglichen, bleibt diesen Betrachtern verborgen. Damit verlieren Angehörige der unteren und mittleren Klassen typischerweise auch das Interesse und die Motivation zur Beschäftigung mit zeitgenössischer Kunst, da diese meist als verstörend und wenig interessant wahrgenommen wird (Bourdieu 1970: 177). Ganz offensichtlich ist die Grundlage für eine angemessene Kunstwahrnehmung, die dem Betrachter auch einen Kunstgenuss verschafft, die Verfügung über ausreichende ästhetische Kompetenz bzw. kulturelles Kapital, was eine grundlegendere Decodierung eines Kunstwerks erlaubt. Typischerweise sind es daher die von Bourdieu als legitimen Künste bezeichneten Werke, die in besonderem Maße zwischen den verschiedenen sozialen Gruppen von Rezipienten differenzieren, da diese nicht unter Verwendung des alltäglichen kulturellen Kapitals genussbringend decodiert werden können:. , Von allen Produkten, die der Wahl der Konsumenten unterliegen, sind die legitimen Kunstwerke die am stärksten klassifizierenden und Klasse verleihenden, [...]“ (Bourdieu 1982: 36). Die Musik stellt für Bourdieu die reinste und legitimste Form der Kunst dar, hier müssten sich daher auch die stärksten Klassifikationswirkungen finden lassen (Bourdieu 1982: 42; Rössel / Hackenbroch / Göllnitz 2002).

In Bourdieus Ausführungen über ästhetische Kompetenz werden verschiedene Quellen von kulturellem Kapital diskutiert. ${ }^{2}$ Als erste Quelle nennt er die wiederholte Beschäftigung mit spezifischen Kunstwerken, die allerdings nur ein intuitives Verständnis von kulturellen Objekten ermöglicht (Bourdieu 1970: 182 f). Dagegen ermöglicht die im Kontext des Bildungssystems als zweitgenannter Quelle erworbene ästhetische Kompetenz ein bewusstes Erfassen von Kunstwerken. Zudem ist diese ästhetische Kompetenz auf verschiedene kulturelle Felder übertragbar. Während aus der intensiven Beschäftigung mit einem kulturellen Feld offensichtlich nur ein feldspezifisches kulturelles Kapital resultiert, wird im schulischen Kontext eine grundsätzliche Haltung zu Kunstwerken entwickelt, die auf ganz unterschiedliche Bereiche übertragbar ist (Bourdieu 1970: 185ff). Man könnte hier im Gegensatz zum feldspezifischen kulturellen Kapital von einem generalisierten kulturellen Kapital sprechen. Auch die dritte von Bourdieu genannte Quelle von kulturellem Kapital, das Elternhaus, erzeugt eine solche übertragbare kulturelle Kompetenz. Das kulturelle Klima im Herkunftshaushalt macht Kinder und Jugendliche schon früh mit einer spezifischen ästhetischen Einstellung vertraut, die den Effekt der schulischen Ausbildung noch einmal verstärkt (Bourdieu 1970: 192 f).

An dieser Stelle wird deutlich, dass in Bourdieus Theorie zwei zentrale Unterschiede in den Rezeptionsformen von Personen aus unterschiedlichen sozialen Klassen formuliert werden. Einerseits erwartet er bei Akteuren aus den oberen sozialen Klassen, die mit schulischem kul-

2 Die Begriffe ,inkorporiertes kulturelles Kapital“ und ,ästhetische Kompetenz“ verwende ich im Kontext dieses Aufsatzes synonym. Bei Bourdieu stellt der zweite Begriff eine Teilmenge des ersten dar. 
turellem Kapital gut ausgestattet sind, eine stärkere Präferenz für Werke der legitimen Kultur. Zweitens vermutet Bourdieu aber nicht nur einen Unterschied in den ästhetischen Vorlieben der Gruppen, sondern auch eine Divergenz in den Formen der Rezeption. Personen mit einer großen ästhetischen Kompetenz sollten zu einer weniger oberflächlichen Rezeption von kulturellen Werken neigen. Diese Unterschiede sollten vor allem in den am stärksten klassifizierenden Formen der legitimen Kultur, also vor allem der Musik, zum Ausdruck kommen.

Die kritische Diskussion von Bourdieus Theorie der kulturellen Reproduktion hat die Beschränkungen einer allein auf bestimmte Inhalte ausgerichteten Interpretation und Operationalisierung seiner Theorie deutlich gemacht. Es ist zu berücksichtigen, dass im Gegensatz zum größten Teil der bisherigen Forschung der Fokus nicht mehr allein auf die unterschiedlichen ästhetischen Präferenzen von Personen gelegt werden sollte, sondern auf die verschiedenen Rezeptionsformen, die Akteure mit unterschiedlicher ästhetischer Kompetenz an den Tag legen. In der hier vorgestellten empirischen Studie über die Rezeption von Opernmusik können genau diese Punkte aufgegriffen werden. Diese soll systematisch die verschiedenen Formen der Rezeption von hochkultureller Musik bestimmen und den Einfluss des kulturellen Kapitals auf das Wie der Konsumtion und Rezeption analysieren. Auf der Grundlage der hier präsentierten Skizze von Bourdieus Theorie der Kunstrezeption lassen sich dabei folgende Hypothesen entwickeln, die in der empirischen Studie geprüft werden können. In den Hypothesen wird unter einer decodierenden Rezeption im Sinne von Bourdieu eine Wahrnehmung und Aufnahme der Musik verstanden, die nicht bei Oberflächenmerkmalen stehen bleibt, sondern das Kunstwerk in seinen formalen und stilistischen Aspekten analysiert.

$\mathrm{H}_{1}$ : Je häufiger sich eine Person mit hochkultureller Musik beschäftigt, desto stärker wird sie diese decodierend rezipieren.

$\mathrm{H}_{2}$ : Je höher die Ausstattung einer Person mit feldspezifischem kulturellem Kapital, desto stärker wird sie Musik decodierend rezipieren.

$\mathrm{H}_{3}$ : Je höher die Bildung einer Person, desto stärker wird sie Musik decodierend rezipieren.

$\mathrm{H}_{4}$ : Je höher das kulturelle Kapital im Elternhaus einer Person, desto stärker wird sie Musik decodierend rezipieren.

\section{Daten und Methode}

Die hier verwendeten Daten entstammen einer Umfrage unter 1170 Befragten in einer Oper einer ostdeutschen Großstadt. Diese wurden im Frühjahr 2005 nach einem systematischen Zufallsverfahren unter den Besuchern von zwanzig Veranstaltungen (14 Opern- und 6 Ballettaufführungen) ausgesucht. Es wurde darauf geachtet, dass die Verteilung der Fragebögen an Werktagen und Wochenenden, in Ballett- und Opernaufführungen sowie bei Premierenund Repertoireveranstaltungen deren Besucherzahlen im Gesamtpublikum im Verlauf eines Jahres entspricht. Die Befragung wurde von geschulten studentischen Mitarbeitern durchgeführt, die die Opernbesucher nach einem Abzählprinzip angesprochen haben, um Selbstselektionseffekte zu vermeiden. Die Befragung fand vor Beginn der Oper und in der Pause statt. Von den 2195 verteilten Fragebögen wurden $61 \%$ zurückgegeben, insgesamt wurden $53 \%$ fast vollständig ausgefüllt. Von den Befragten verfügten 40,9\% über eine Abonnementvariante, ${ }^{3} 59 \%$ waren Frauen, 61,3\% verfügten über einen Fachhochschul- oder Hochschulabschluss. Der Altersdurchschnitt der befragten Opernbesucher lag bei 50,6 Jahren. Diese Angaben zum befragten Publikum verdeutlichen, dass die Stichprobe eine höchst selektive Zusammensetzung aufweist, die sich allerdings nicht systematisch vom Publikum anderer Veranstaltungen und Einrichtungen im Bereich der klassischen Musik unterscheidet (Rössel / Hackenbroch / Göllnitz 2002, 2005). Allerdings ist mit dieser Stichprobenzusammensetzung ein

3 Die Oper bietet eine Vielzahl von unterschiedlichen Abonnements an, die sich nur zum Teil mit einem klassischen Abonnement decken. 
methodisches Problem verbunden: will man die Rezeption von klassischer Musik bzw. Opernmusik analysieren, müssen selbstverständlich Personen befragt werden, die diese Musikgenres tatsächlich hören. Personen, die diese Musikrichtungen nicht schätzen bzw. nicht rezipieren, können typischerweise auch nur in beschränktem Maße Auskunft über ihre Wahrnehmung dieser Musik geben. Insofern bietet sich eine Publikumsumfrage in einem klassischen Konzerthaus oder in einer Oper für eine solche Untersuchung an. Darüber hinaus sind Besucherumfragen im Vergleich zu Bevölkerungsumfragen weniger anfällig für Antwortmuster, die auf sozialer Erwünschtheit basieren (Reuband 2007). Eine Publikumsumfrage hat allerdings den Nachteil, dass man es mit einem stark selektiven Publikum zu tun hat, das im Durchschnitt stark an klassischer Musik interessiert ist und einen sehr hohen Anteil hoch gebildeter Personen aufweist. Dies muss auch bei der Interpretation der Ergebnisse berücksichtigt werden. Um die vorliegende Forschungsfrage aber überhaupt beantworten zu können, ist die empirische Vorgehensweise trotz aller Probleme angemessen. Darüber hinaus muss bei der Interpretation der Ergebnisse berücksichtigt werden, dass die Umfrage in Ostdeutschland stattgefunden hat. Zwar zeigt sich in den vorliegenden Studien zum Vergleich der Milieu- und Lebensstillandschaft in West- und Ostdeutschland eine große Übereinstimmung, aber in den meisten Studien ist ersichtlich, dass die Lebensstile in Ostdeutschland weniger stark durch die Bildung von Personen geprägt sind als in Westdeutschland, was bei der Interpretation der Resultate in Rechnung zu stellen ist (Lechner 2002; Rössel 2004: 103).

Um die Rezeption von klassischer bzw. Opernmusik in der Befragung zu erheben, konnte auf eine Itembatterie aus der Musikpsychologie zurückgegriffen werden, die von Behne (1986) entwickelt wurde und seitdem in einer ganzen Reihe von Studien verwendet wurde (Lehmann 1993, 1994; Behne 1997, 2001). Behne hat diese empirischen Indikatoren entwickelt, um sich von einer stark normativen Perspektive auf das Hören von Musik abzugrenzen, wie sie z. B. von Adorno entwickelt wurde (1976). Ähnlich wie Bourdieu in seinen Ausführungen über Kunstrezeption favorisiert Adorno einen intellektuellen, strukturierten Zugang zur Musik und disqualifiziert anders geartete Zugänge als minderwertig. Behnes Indikatorenbatterie versucht dagegen erstmalig empirisch zu erfassen, wie Menschen tatsächlich Musik erfassen und rezipieren. Daraus wurden 24 Items (vgl. Tabelle 1 im Anhang) ausgewählt und speziell für das Hören von Opernmusik angepasst. Im Gegensatz zu einer Besucherstudie über Musikrezeption, die Roose (2008) in Belgien durchgeführt hat, konnte hier also auf ein vielfach geprüftes und validiertes Messinstrument zurückgegriffen werden. Da sich die von Roose erhobenen Daten in ihrem Inhalt mit der Itembatterie von Behne überschneiden, können dessen Ergebnisse teilweise zum Vergleich herangezogen werden. Auch die verschiedenen Arten des kulturellen Kapitals und ihre Quellen wurden durch eine Reihe von Indikatoren operationalisiert. So weist Bourdieu darauf hin, dass schon die wiederholte Beschäftigung mit Kunstwerken zu einem besseren Verständnis beiträgt, das allerdings auf das jeweils spezifische Genre beschränkt bleibt. Daher wurde für die Befragten ihre Besuchshäufigkeit von verschiedenen Formen von Konzerten erhoben (Operette, Musical, Oper, Ballett, klassisches Konzert im Konzerthaus). Auf der Basis einer unrotierten Faktorenanalyse (vgl. Tabelle 2 im Anhang) können hier zwei Dimensionen unterschieden werden: einerseits die Partizipation in Veranstaltungen eines „mittleren“ Geschmacksniveaus (vor allem Operette und Musical) und die Teilnahme an Hochkulturveranstaltungen (vor allem Oper, Ballett und klassisches Konzert). Darüber hinaus können noch drei weitere Variablen zur Messung des musikspezifischen kulturellen Kapitals verwendet werden: erstens wurde das opernspezifische kulturelle Kapital über die Lektüre von Opernfachliteratur und Opernzeitschriften operationalisiert. Zweitens wurde eine unrotierte Faktorenanalyse auf der Grundlage von fünf Items (Hat der Befragte ein Musikinstrument erlernt, hat er eine Musikschule besucht, musiziert der Befragte heute noch, waren die Eltern des Befragten musikinteressiert, war der Befragte schon als Kind in der Oper oder in einem klassischen Konzert) durchgeführt, die zu zwei deutlich separierten Dimensionen geführt hat (vgl. Tabelle 3 im Anhang). Einerseits führt sie zu einer Komponente, die auch das musik- 
spezifische kulturelle Kapital erfasst, auf ihr laden vor allem Indikatoren, die die aktive eigenständige Beschäftigung mit Musik betreffen, andererseits findet sich eine Dimension, die vor allem den Erwerb von kulturellem Kapital im Elternhaus anzeigt und damit die dritte von Bourdieu genannte Quelle kulturellen Kapitals abdeckt. Im Hinblick auf die schulische Bildung können unter den Besuchern nur grobe Differenzierungen vorgenommen werden, da im Publikum der Oper die hoch gebildeten Bevölkerungsgruppen stark überrepräsentiert sind. Daher wird hier nur zwischen Personen mit abgeschlossener Sekundarschulausbildung (Abitur), Personen mit abgeschlossener Tertiärausbildung (Hochschulabschluss) und allen anderen unterschieden. Dies wird durch zwei Dummyvariablen operationalisiert. Damit können die verschiedenen Formen von kulturellem Kapital und ihre Quellen in dieser Untersuchung in ausgesprochen differenzierter Form erfasst werden und systematisch in ihrem Einfluss auf die verschiedenen Rezeptionsweisen von Opernmusik betrachtet werden, wie er in den Hypothesen 1 bis 4 formuliert wurde. Um auch andere Einflüsse auf die Musikrezeption zu berücksichtigen, wurden noch vier Kontrollvariablen berücksichtigt. Erstens wurde in Betracht gezogen, ob die Befragten die Veranstaltung alleine besucht haben, da dies für ein besonders ernsthaftes und konzentriertes Interesse an der Musik spricht. Zweitens können mit dem Geschlecht, dem Alter und dem Einkommen drei weitere Variablen berücksichtigt werden, die sich im Bereich der Kunstrezeption und der Lebensstilforschung als besonders erklärungskräftig erwiesen haben (Otte 2004; Rössel 2005; van Eijck / Bargeman 2004). Insofern kann in dieser Studie der Einfluss der verschiedenen Arten kulturellen Kapitals auf die Musikrezeption unter Berücksichtigung einer Reihe von Kontrollvariablen systematisch geprüft werden.

\section{Empirische Resultate}

In der empirischen Analyse der vorliegenden Daten aus der Besucherumfrage wurden vor allem zwei Fragen untersucht, die zentral für die soziologische Theorie der Kunstrezeption von Bourdieu sind: erstens welche verschiedenen Typen der Musikrezeption sich auf der Grundlage der Indikatorenbatterie von Behne (1986) für das Opernpublikum ergeben und zweitens welchen Einfluss die verschiedenen Arten und Quellen des kulturellen Kapitals der Befragten auf die Ausprägung der unterschiedlichen Rezeptionsformen haben. Damit kann auch die Gültigkeit der Hypothesen 1 bis 4 empirisch geprüft werden.

Wenden wir uns an dieser Stelle der ersten Frage zu: welche Rezeptionsweisen von Opernmusik ergeben sich auf der Grundlage der Indikatorenbatterie von Behne (1986)? Dazu wurde eine unrotierte Faktorenanalyse mit allen 24 berücksichtigten Items durchgeführt. Diese führt zu sechs relativ klar von einander abgegrenzten Dimensionen. Auf der ersten Dimension (Gefühle) laden vor allem Items, die auf einen gefühlsmäßigen und stimmungsorientierten Zugang zur Musik verweisen. Dagegen taucht als zweite Komponente (Analyse) eine Rezeptionsform auf, die dem von Adorno favorisierten intellektuellen Hören bzw. der von Bourdieu als angemessene Decodierung bezeichneten Form der Kunstrezeption entspricht. Diese Dimension kann verwendet werden, um die Hypothesen 1 bis 4 systematisch zu prüfen. Auf ihr laden Items, die ein bewusstes Analysieren des musikalischen Aufbaus und der musikalischen Form zum Gegenstand haben. Ganz anders geartet ist dann wiederum die dritte Hörweise, die sich in der Faktorenanalyse herauskristallisiert (Eskapismus). Hier deutet sich eine Form des eskapistischen Musikgenusses an, der zwar auf der einen Seite auch durch einen emotionalen Zugang zur Musik charakterisiert ist, auf der anderen Seite aber die Musik vor allem als einen Ausweg aus der Wirklichkeit begreift und damit den Charakter von Weltflucht hat. Eine vierte Form der Musikrezeption (Oberflächlich) begreift die Musik vor allem als Hintergrund und wendet sich zum Teil anderen Gedanken zu bzw. ist nur sehr begrenzt tatsächlich auf die Musik konzentriert. Die fünfte Dimension (Vegetativ) scheint auf den ersten Blick kaum in das Szenario einer Opernaufführung zu passen, da hier die Musik vor allem vegetativ wahrgenommen 
wird. Auf dieser Komponente laden Items, die auf die körperliche Beteiligung der Befragten verweisen. Schließlich ergibt sich als sechste Form der Musikrezeption (Konzentriert) eine bewusst konzentrierte, die vor allem die Musik und den Gesang im Fokus hat. Den hier gefundenen sechs Dimensionen kommt besondere Validität zu, da sie nahezu exakt mit den von Behne festgestellten Dimensionen übereinstimmen (Behne 1997: 147). Lediglich die hier als „Gefühle“ bezeichnete Form der Musikrezeption wird bei Behne in zwei Unterdimensionen zerlegt, eine mit der Bezeichnung „Emotional“, die andere mit dem Namen „Sentimental“. Ansonsten ergibt sich eine fast vollständige Übereinstimmung. ${ }^{4}$ Berücksichtigt man die Tatsache, dass Behne eine sozial heterogene Stichprobe von Jugendlichen befragt hat, dann ist die Übereinstimmung mit den festgestellten Dimensionen der Musikrezeption bei älteren und im Durchschnitt hoch gebildeten Opernbesuchern geradezu verblüffend.

Aus dieser empirischen Analyse von Formen des Musikhörens wird deutlich, dass die Realität der Kunstrezeption vielgestaltiger ist als Bourdieus Theorie der Kunstrezeption es vorsieht. Lediglich die Dimension „Analyse“ entspricht vollständig dem Gegensatz zwischen einem oberflächlichen Hören auf der einen Seite und einem intellektuellen, decodierenden Hören auf der anderen Seite. Die anderen Hörweisen lassen sich nicht eindeutig in der von Bourdieu theoretisch begründeten Spannbreite von Rezeptionsformen von Kunst einordnen. Damit kann als erstes Zwischenergebnis an dieser Stelle festgehalten werden, dass eine vollständige soziologische Theorie der Kunstrezeption auch die verschiedenen Formen der Rezeption angemessen erfassen und theoretisch erklären muss, was für Bourdieus Theorie offensichtlich nicht gilt. Gleichzeitig gibt es aber eine Dimension der Musikrezeption, die dem von Bourdieu diskutierten decodierenden Verständnis entspricht, so dass die aus seiner Theorie der Kunstrezeption abgeleiteten Hypothesen 1 bis 4 hier empirisch geprüft werden können.

4 Behne (1997) kommt in seiner Studie auf neun Dimensionen. Die Items der zwei hier nicht auftauchenden Dimensionen (Associative, Stimulative) wurden in dieser Studie nicht berücksichtigt, da sie für das Opernpublikum als weniger relevant betrachtet wurden. 
Tabelle 1: Struktur der Musikrezeption

\begin{tabular}{|c|c|c|c|c|c|c|}
\hline & $\begin{array}{l}\text { Ge- } \\
\text { fühle }\end{array}$ & $\begin{array}{c}\text { Analy- } \\
\text { se }\end{array}$ & $\begin{array}{c}\text { Eskapis- } \\
\text { mus }\end{array}$ & $\begin{array}{l}\text { Oberfläch- } \\
\text { lich }\end{array}$ & $\begin{array}{c}\text { Vegeta- } \\
\text { tiv }\end{array}$ & $\begin{array}{c}\text { Konzen- } \\
\text { triert }\end{array}$ \\
\hline Bad in Klängen & 0,74 & --- & $\begin{array}{l}-- \\
\end{array}$ & --- & $\begin{array}{l}-- \\
\end{array}$ & --- \\
\hline mit Gefühl hören & 0,63 & --- & --- & --- & --- & --- \\
\hline $\begin{array}{l}\text { körperliche Wirkungen } \\
\text { spüren }\end{array}$ & 0,68 & --- & --- & --- & --- & --- \\
\hline $\begin{array}{l}\text { Gefühle und Stimmungen } \\
\text { in der Musik wiederfinden }\end{array}$ & 0,63 & --- & --- & --- & --- & --- \\
\hline möchte am liebsten weinen & 0,53 & --- & --- & --- & --- & --- \\
\hline Musik geht unter die Haut & 0,78 & --- & --- & --- & --- & --- \\
\hline $\begin{array}{l}\text { sich ganz der Musik hinge- } \\
\text { ben }\end{array}$ & 0,52 & --- & 0,42 & --- & --- & --- \\
\hline $\begin{array}{l}\text { Rhythmus hält mich gefan- } \\
\text { gen }\end{array}$ & 0,52 & --- & 0,31 & --- & --- & --- \\
\hline $\begin{array}{l}\text { Gefühle werden in der Mu- } \\
\text { sik ausgedrückt }\end{array}$ & 0,35 & 0,41 & 0,44 & --- & --- & --- \\
\hline $\begin{array}{l}\text { Themen und Melodien } \\
\text { verfolgen }\end{array}$ & 0,49 & 0,45 & --- & --- & --- & --- \\
\hline $\begin{array}{l}\text { versuche, Formaufbau zu } \\
\text { verstehen }\end{array}$ & --- & 0,76 & --- & --- & --- & --- \\
\hline $\begin{array}{l}\text { achte auf Gestaltung durch } \\
\text { Komponisten }\end{array}$ & --- & 0,79 & --- & --- & --- & --- \\
\hline $\begin{array}{l}\text { achte auf Änderungen der } \\
\text { Tonart }\end{array}$ & --- & 0,77 & --- & --- & --- & --- \\
\hline $\begin{array}{l}\text { versuche die Opernart er- } \\
\text { kennen }\end{array}$ & --- & 0,50 & --- & --- & 0,40 & --- \\
\hline $\begin{array}{l}\text { achte auf die Qualität der } \\
\text { Musiker }\end{array}$ & --- & 0,60 & --- & --- & --- & 0,41 \\
\hline $\begin{array}{l}\text { Musik soll mich auf andere } \\
\text { Gedanken bringen }\end{array}$ & --- & --- & 0,53 & --- & 0,31 & --- \\
\hline fühle mich weniger einsam & --- & --- & 0,73 & --- & --- & --- \\
\hline träume ich am liebsten & --- & --- & 0,54 & 0,44 & --- & --- \\
\hline höre oft nur mit einem Ohr & --- & --- & --- & 0,84 & --- & --- \\
\hline $\begin{array}{l}\text { habe irgendwelche ande- } \\
\text { ren Gedanken }\end{array}$ & -- & --- & --- & 0,84 & --- & --- \\
\hline möchte ich mitsummen & --- & --- & --- & --- & 0,79 & --- \\
\hline möchte ich mich bewegen & --- & --- & --- & --- & 0,75 & --- \\
\hline $\begin{array}{l}\text { Musik steht im Vorder- } \\
\text { grund }\end{array}$ & --- & -- & --- & -- & --- & 0,74 \\
\hline $\begin{array}{l}\text { Gesang steht im Vorder- } \\
\text { grund }\end{array}$ & --- & --- & --- & --- & --- & 0,72 \\
\hline
\end{tabular}

\begin{tabular}{lllllll}
\hline Erklärte Varianz in \% & 23,53 & 10,48 & 7,18 & 5,30 & 5,25 & 4,20 \\
\hline
\end{tabular}

* Faktorladungen kleiner als 0,3 werden nicht angezeigt.

Im zweiten Schritt der Untersuchung soll nun betrachtet werden, ob die verschiedenen Indikatoren für das kulturelle Kapital auch unter Berücksichtigung von Kontrollvariablen einen Einfluss auf die Ausprägung der musikalischen Hörweisen der Befragten haben wie er in den Hypothesen 1 bis 4 formuliert wurde. Allerdings soll hier nicht allein die Rezeptionsdimension Analyse betrachtet werden, sondern auch untersucht werden, wie groß der Einfluss des kulturellen Kapitals auf die anderen Dimensionen der Musikwahrnehmung ist. Dazu wurden die sechs Dimensionen aus der in Tabelle 1 präsentierten Faktorenanalyse als abhängige Variablen in sechs linearen Regressionsmodellen betrachtet. Diese sind in Tabelle 2 dargestellt. Ein Blick auf das $\mathrm{R}^{2}$ der verschiedenen statistischen Modelle zeigt, dass nur in einem einzigen Fall eine zufriedenstellende Varianzaufklärung gelingt. Nur die analytische Hörweise kann durch die 
berücksichtigten unabhängigen Variablen in einem akzeptablen Maß erklärt werden. Dieses Ergebnis deckt sich in erstaunlichem Maße mit den empirischen Resultaten von Roose (2008: 246). Dies deutet darauf hin, dass die Rezeption von Musik mit konventionellen soziologischen Variablen nur beschränkt erklärt werden kann. Ein genauerer Blick auf die zweite Spalte in Tabelle 2 zeigt, dass die Ergebnisse für die analytische Hörweise überwiegend der soziologischen Theorie der Kunstrezeption von Bourdieu und den daraus abgeleiteten Hypothesen entsprechen. Personen, die häufig Hochkulturveranstaltungen besuchen, die über viel opernspezifisches Kapital verfügen und selbst aktiv Musik betreiben, neigen in stärkerem Maße zu einer analytischen Hörweise. Keinen statistisch signifikanten Einfluss hat wider Erwarten die schulische Bildung, was aber auch auf die Überrepräsentation von hochgebildeten Personen im Opernpublikum zurückzuführen sein mag und das kulturelle Klima im Elternhaus. In dieser Dimension der Musikrezeption, die explizit in Bourdieus Theorie thematisiert wird, können also nur zwei von vier Hypothesen bestätigt werden. Welche Rolle spielt nun aber das kulturelle Kapital in seinen verschiedenen Operationalisierungsformen für die anderen Typen des $\mathrm{Mu}-$ sikhörens? Für die schulische Bildung als Operationalisierung des generalisierten kulturellen Kapitals findet sich lediglich ein einziger statistisch signifikanter Effekt. Personen mit einer abgeschlossenen Hochschulausbildung neigen etwas stärker zur emotionalen Hörweise (Gefühle) als die Vergleichsgruppen. Dieses spezifische Ergebnis deutet darauf hin, dass auch ein gefühlsmäßiges Verständnis von Musik bestimmte Voraussetzungen hinsichtlich des Erfassens und des Verstehens von Musik hat. Doch insgesamt wird deutlich, dass die schulische Bildung keine besonders wichtige Einflussgröße ist. Dies dürfte aber im Zusammenhang mit der spezifischen Stichprobe in dieser Untersuchung stehen, die im Hinblick auf das schulische kulturelle Kapital relativ homogen zusammengesetzt ist. Allerdings zeigt sich auch für das aktive Musizieren als einer Form des musikspezifischen kulturellen Kapitals nur ein statistisch signifikanter Effekt über den oben schon genannten hinaus. Personen, die aktiv Musik betreiben, neigen weniger zu einer eskapistischen Herangehensweise an Musik. Ein vergleichbares Resultat lässt sich für das kulturelle Klima im Elternhaus feststellen: Dieses hat wiederum nur einen statistisch signifikanten positiven Effekt auf das konzentrierte Hören von Musik. Das opernspezifische kulturelle Kapital hat über den oben diskutierten Effekt auf die analytische Hörweise hinaus auch nur einen weiteren bedeutsamen Einfluss auf das konzentrierte Hören von Musik. Die meisten statistisch signifikanten Parameter unter den verschiedenen Indikatoren für das kulturelle Kapital finden sich für den Besuch von Hochkulturveranstaltungen. Neben dem schon genannten Einfluss auf das analytische Hören finden sich ein weiterer positiver Einfluss auf das gefühlsmäßige Hören und ein negativer Effekt auf das oberflächliche Hören. Insgesamt ist also das Resultat im Hinblick auf die Erklärungskraft des kulturellen Kapitals für die verschiedenen Formen der Musikrezeption recht bescheiden. Die analytische Hörweise, die in hohem Maße den theoretischen Konstrukten der soziologischen Theorie der Kunstrezeption von Bourdieu entspricht, kann noch relativ gut durch die Ausstattung mit kulturellem Kapital erklärt werden, wenngleich nur zwei der vier Hypothesen Bestätigung finden. Die anderen Formen der Musikrezeption können aber durch die hier verwendeten, schon relativ vielgestaltigen, Indikatoren für kulturelles Kapital kaum erklärt werden. Als besonders relevant erweist sich vor allem die wiederholte Beschäftigung mit Musik durch den Besuch von Hochkulturveranstaltungen. Die regelmäßigen Besucher unterscheiden sich also in ihrer Musikrezeption von den seltenen Besuchern (vgl. auch Roose 2008: 246). Dies spricht dafür, dass Bourdieus Theorie die verschiedenen Formen der Kunstrezeption nicht nur unzulässig auf eine Dimension verkürzt, sondern auch die erklärenden Faktoren nur ausschnitthaft thematisiert. Interessanterweise ist das Lebensalter die Variable mit den stärksten statistisch signifikanten Effekten: es hat einen positiven Einfluss auf das konzentrierte und das eskapistische Hören und einen negativen auf das gefühlsmäßige und oberflächliche Hören. 
Tabelle 2: Determinanten der Musikrezeption

\begin{tabular}{lcccccc}
\hline & Analyse & $\begin{array}{c}\text { Konzen- } \\
\text { triert }\end{array}$ & Gefühle & $\begin{array}{c}\text { Eskapis- } \\
\text { mus }\end{array}$ & $\begin{array}{c}\text { Vegeta- } \\
\text { tiv }\end{array}$ & $\begin{array}{c}\text { Oberfläch- } \\
\text { lich }\end{array}$ \\
\hline Single & 0,002 & 0,021 & $-0,002$ & 0,011 & 0,011 & $-0,026$ \\
Mittlere Bildung & $(0,048)$ & $(0,603)$ & $(-0,049)$ & $(0,299)$ & $(0,305)$ & $(-0,744)$ \\
Hohe Bildung & $-0,069$ & $-0,032$ & 0,088 & $-0,077$ & $-0,020$ & 0,042 \\
& $(-1,492)$ & $(-0,652)$ & $(1,801)$ & $(-1,553)$ & $(-0,390)$ & $(0,875)$ \\
Alter & $-0,044$ & $-0,066$ & $0,097^{*}$ & $-0,028$ & 0,000 & 0,011 \\
& $(-0,942)$ & $(-1,331)$ & $(1,976)$ & $(-0,568)$ & $(0,006)$ & $(0,220)$ \\
Einkommen & 0,053 & $0,173 * *$ & $-0,095^{*}$ & $0,089^{*}$ & $-0,058$ & $-0,287^{* *}$ \\
& $(1,451)$ & $(4,429)$ & $(-2,460)$ & $(2,247)$ & $(-1,462)$ & $(-7,511)$ \\
Geschlecht & 0,030 & $-0,018$ & $0,081^{*}$ & $-0,077^{*}$ & 0,014 & $-0,039$ \\
(Referenz: männlich) & $(0,826)$ & $(-0,458)$ & $(2,127)$ & $(-1,986)$ & $(0,365)$ & $(-1,043)$ \\
Aktives Musizieren & $-0,100^{* *}$ & $-0,025$ & $0,162^{* *}$ & 0,034 & 0,027 & 0,011 \\
& $(-2,987)$ & $(-0,684)$ & $(4,555)$ & $(0,940)$ & $(0,748)$ & $(0,326)$ \\
Kulturelles Klima im El- & $0,271 * *$ & 0,002 & 0,043 & $-0,091 *$ & 0,042 & $-0,010$ \\
ternhaus & $(7,964)$ & $(0,049)$ & $(1,190)$ & $(-2,469)$ & $(1,137)$ & $(-0,292)$ \\
Opernspezifisches Kapi- & $(1,723)$ & $0,069^{*}$ & 0,013 & $-0,012$ & 0,019 & $-0,032$ \\
tal & $0,245^{* *}$ & $0,076 *$ & $(0,377)$ & $(-0,328)$ & $(0,541)$ & $(-0,943)$ \\
Besuch von Hochkultur- & $(7,427)$ & $(2,155)$ & $(1,3076$ & $-0,011$ & 0,030 & $-0,002$ \\
veranstaltungen & $0,114 * *$ & 0,046 & $0,122^{* *}$ & $(-0,315)$ & $(0,821)$ & $(-0,045)$ \\
Besuch von Veranstal- & $(3,373)$ & $(1,284)$ & $(3,405)$ & $(-0,242)$ & $(-1,868)$ & $(-2,584)$ \\
tungen ,mittleren“ Ge- & $-0,14$ & 0,003 & $-0,59$ & $-0,011$ & 0,051 & $-0,037$ \\
schmacksniveaus & $(-0,435)$ & $(0,076)$ & $(-1,694)$ & $(-0,415)$ & $(1,425)$ & $(-1,083)$ \\
Korrigiertes R ${ }^{2}$ & & & & & & \\
N & 0,159 & 0,039 & 0,056 & 0,014 & 0,001 & 0,092 \\
\hline
\end{tabular}

$* \mathrm{p}<0,01, * * \mathrm{p}<0,001$. Dargestellt sind die standardisierten Regressionskoeffizienten sowie die t-Werte (in Klammern).

\section{Zusammenfassung und Diskussion}

Der Ausgangspunkt für die vorliegende empirische Studie über kulturelles Kapital und Musikrezeption war die Kritik an Bourdieus Theorie der kulturellen Reproduktion von sozialen Klassen. Vor allem zwei Punkte wurden hervorgehoben: einerseits wurde häufig kritisiert, dass die Theorie der Populärkultur eine zu geringe Aufmerksamkeit widmet, andererseits wurde in Frage gestellt, dass Unterschiede in der Ausstattung mit kulturellem Kapital tatsächlich so relevant für Divergenzen in den Rezeptionsformen sind. Der zweite dieser Kritikpunkte wurde hier am Beispiel der Opernrezeption systematisch empirisch untersucht. Um diese Probleme differenziert zu behandeln, wurde in einem ersten Schritt Bourdieus Theorie der Kunstrezeption skizziert, so dass verschiedene Quellen kulturellen Kapitals in ihrer Relevanz für den Geschmack und die jeweilige Rezeptionsform unterschieden werden konnten.

In der vorliegenden Studie wurde empirisch geprüft, ob die verschiedenen Arten von kulturellem Kapital mit ihren unterschiedlichen sozialen Quellen tatsächlich die erwarteten Effekte auf die Opernrezeption haben. Dabei wurde erstens deutlich, dass die Formen der Rezeption von Musik deutlich vielfältiger sind als in Bourdieus Theorie vorgesehen. Neben der von ihm thematisierten Rezeptionsdimension, die sich zwischen einem oberflächlich und einem analytisch-decodierenden Verständnis von Kunst aufspannt, konnten noch fünf weitere Hörweisen von Musik ausgemacht werden. Da diese mit den von Behne (1997) festgestellten Rezeptionsformen von Musik in hohem Maße korrespondieren, kommt diesen Ergebnissen 
eine hohe Validität zu. Dies stellt überkommene Vorstellungen über die Unterschiede von Hoch- und Populärkultur in Frage. Während der Rezeption von Populärkultur zumeist eskapistische und affektive Motive und Rezeptionsweisen zugeschrieben wurden, wurde die Hochkultur typischerweise als eine Domäne einer konzentrierten und vornehmlich intellektuellen Wahrnehmung der Künste betrachtet (Levine 1990; DiMaggio 1982 b). Die vorliegenden Ergebnisse verdeutlichen, dass dieser Unterschied keineswegs so deutlich ausgeprägt ist und dass auch im Bereich der Opernmusik, als einem prototypischen Beispiel für die klassische Hochkultur, oberflächliche, emotionale und eskapistische Zugangsweisen im Publikum verbreitet sind. Zweitens wurde aber deutlich, dass die Ausstattung mit kulturellem Kapital, die hier mit einer Vielzahl von Indikatoren erhoben wurde, nur die analytische Rezeption von Musik angemessen erklären konnte. Dagegen hat das kulturelle Kapital nur unwesentliche Relevanz für die anderen Formen der Musikrezeption. Darüber hinaus wurde auch deutlich, dass vor allem die musikspezifischen Formen des kulturellen Kapitals besonders erklärungskräftig waren, während die generalisierten Formen des kulturellen Kapitals, die in Elternhaus und Schule erworben werden, eine geringere Bedeutung haben. Allerdings könnte dies auf die Tatsache zurückzuführen sein, dass im Publikum der Oper eine starke Überrepräsentation von Personen mit hohem schulischem kulturellem Kapital existiert. Zudem muss hier in Rechnung gestellt werden, dass für Ostdeutschland wiederholt festgestellt wurde, dass Lebensstile und Hochkulturkonsum in geringerem Maße durch die Bildung beeinflusst werden. Diese Vermutung wird durch die Ergebnisse von Roose (2008) unterstützt, der in einer Besucheranalyse in Belgien eine stärkere Prägung der Musikrezeption durch die Bildung feststellt. Insofern kann der geringe Einfluss der Bildung in der hier vorgelegten Studie auch den Spezifika der ostdeutschen Sozialstruktur geschuldet sein.

Zusammenfassend kann einerseits festgestellt werden, dass die in Bourdieus Theorie der Kunstrezeption angesprochenen theoretischen Zusammenhänge in dieser Studie teilweise empirisch bestätigt werden konnten. Es wurde aber andererseits deutlich, dass seine Theorie nur einen partiellen Charakter hat, da sie nur eine von mehreren Rezeptionsformen betrachtet und auch diese eine Rezeptionsform nur in Grenzen erklären kann. Insofern spricht diese Studie zwar gegen die These von Halle (1993), dass die Ausstattung mit kulturellem Kapital in verschiedenen sozialen Klassen keinen Einfluss auf die Rezeption von Kunstwerken hat. Allerdings zeigt sie auch, dass man gegen die Interpretation von Holt $(1997,1998)$ mit einer nur auf Bourdieus Theorie der Kunstrezeption gestützten Analyse die Vielfalt und Reichweite der möglichen Rezeptionsformen und Aneignungsweisen von kulturellen Gütern deutlich unterschätzt sowie deren Prägung durch das klassenspezifische kulturelle Kapital überschätzt.

In der vorliegenden Studie ist zudem eine weitere empirische Begrenzung von Bourdieus Theorie der Kunstrezeption deutlich geworden. Die Variable mit den meisten und den stärksten statistisch signifikanten Effekten auf die Musikrezeption ist das Lebensalter. Dieses wird allerdings in Bourdieus Texten über Kunstrezeption kaum behandelt, obwohl es sich immer wieder als eine zentrale Determinante von Geschmack und Rezeption erwiesen hat (Behne 1993: 346; van Eijck 2001; van Eijck / Bargeman 2004; Schulze 1992; Otte 2004; Rössel 2005). Die hier vorgestellten Ergebnisse zeigen, dass die älteren Befragten die Musik deutlich konzentrierter und weniger oberflächlich wahrnehmen. Insgesamt machen die dargestellte theoretische Diskussion und die empirischen Resultate ersichtlich, dass Bourdieus Theorie der Kunstrezeption zwar einen zentralen Ausgangspunkt für eine umfassende Theorie der ästhetischen Rezeption und der ästhetischen Präferenzen darstellt, dass es sich aber bisher nur um eine partielle Theorie handelt, die in verschiedenen Hinsichten ergänzt werden muss. Im Hinblick auf den Zusammenhang von Musikgeschmack und Musikrezeption mit dem Lebensalter bieten sich vor allem zwei Erklärungsmuster an. Erstens kann hier auf kohortenspezifische Prägungen des Musikgeschmacks verwiesen werden. Eine Vielzahl von empirischen Resultaten weist darauf hin, dass vor allem die in der Phase der späten Adoleszenz und des frühen 
Erwachsenenalters präferierte Musik prägend für das gesamte Leben bleibt (North / Hargreaves 2008: 107ff). In weitergehenden Studien konnte demonstriert werden, dass unterschiedlichste Arten von Präferenzen typischerweise in diesem Lebensalter bestimmt werden. Allerdings hilft uns dieser theoretische Ansatz nur wenig bei der Erklärung des Zusammenhangs von Rezeptionsweisen und Alter. Daher sollte an dieser Stelle zweitens auf den Beitrag der sogenannten Informationsästhetik verwiesen werden (North / Hargreaves 2008: 76ff). Diese geht davon aus, dass zwischen der Komplexität von musikalischen Werken und dem subjektiven Gefallen an ihnen ein umgekehrt u-förmiger Zusammenhang besteht, der in einer Reihe von zumeist experimentellen Studien auch demonstriert werden konnte. Sehr einfache und sehr komplexe Werke werden von Hörern eher negativ bewertet, während Stücke mittlerer Komplexität positiv eingeschätzt werden. Die subjektiv wahrgenommene Komplexität eines Musikstücks hängt allerdings von der wiederholten Beschäftigung und Vertrautheit mit einem Werk ab. Insofern werden Personen, die über viele Erfahrungen im Umgang mit komplexen Musikstücken, also z. B. Werken der klassischen Musik, verfügen diese positiver bewerten und einen größeren Hörgenuss erleben. Mit zunehmendem Alter und damit einer umfangreicheren Beschäftigung mit Musik nehmen Personen Stücke subjektiv daher als zunehmend weniger komplex wahr, so dass zunehmend komplexere Werke im Verlauf des Lebens positiv bewertet werden. Damit einher geht dann auch die Fähigkeit zu einem konzentrierteren Hören, das nicht abschweift, während Menschen mit weniger Fähigkeiten einen geringeren Genuss verspüren und komplexen Werken nicht konzentriert folgen können. Die hier nur skizzierte Theorie der Informationsästhetik könnte also eine umfassende Erklärung der hier gefundenen Zusammenhänge zwischen Alter und Musikrezeption bieten. Darüber hinaus könnte man sie auch zur Erklärung der von Bourdieu postulierten Zusammenhänge von Bildung, kulturellem Kapital und Kunstrezeption heranziehen, da auch in seiner Theorie die Fähigkeit zur Entschlüsselung von komplexen Werken im Mittelpunkt steht. Die Informationsästhetik könnte insofern eine zusammenfassende Erklärung der verschiedenen empirischen Resultate ermöglichen. Soziologisch wäre damit allerdings ein veränderter Blick auf die zentralen Strukturen der Gesellschaft verbunden. Das kulturelle Kapital in Bourdieus soziokultureller Klassentheorie ist primär ein Resultat von Sozialisationsprozessen von Menschen in unterschiedlichen sozialen Klassen, also ein Ausdruck der Klassenstruktur der Gesellschaft. Die im Lebensverlauf gewonnene Vertrautheit und Kompetenz im Umgang mit unterschiedlichen Kunstwerken ist dagegen primär der wiederholten und regelmäßigen Partizipation von Personen in einem bestimmten gesellschaftlichen Feld zuzuschreiben. Insofern unterstützen diese empirischen Resultate die differenzierungstheoretische Lesart des Verhältnisses von Feldern und Klassen bei Bourdieu, die Kieserling (2008) jüngst vorgelegt hat. Die Relevanz des Lebensalters in der vorliegenden Studie deutet an, dass die Musikrezeption nicht allein durch das kulturelle Kapital geprägt wird, das aufgrund unterschiedlicher Klassenherkunft erworben wird, sondern auch durch das kulturelle Kapital, dass durch langwierige Partizipation in einem ausdifferenzierten gesellschaftlichen Feld erlernt wird. Insofern sprechen diese Resultate deutlich für eine Aufnahme der theoretischen Diskussion über das Verhältnis von Theorien sozialer Ungleichheit einerseits und Differenzierungstheorien andererseits auch im Feld der Musik- und Kunstsoziologie.

\section{Literatur}

Adorno, Theodor (1976): Introduction to the Sociology of Music, New York.

Aschaffenburg, Karen / Ineke Maas (1997): Cultural and Educational Careers: The Dynamics of Social Reproduction, in: American Sociological Review 62, S. 573-587.

Behne, Klaus-Ernst (1986): Hörertypologien. Zur Psychologie des jugendlichen Musikgeschmacks, Regensburg. 
Behne, Klaus-Ernst (1993): Musikpräferenzen und Musikgeschmack, in: Herbert Bruhn / Rolf Oerter / Helmut Rösing (Hrsg.), Musikpsychologie, Reinbek / Hamburg, S. 339-353.

Behne, Klaus-Ernst (1997): The Development of „Musikerleben” in Adolescence: How and Why Young People Listen to Music, in: Irene Deliége / John Sloboda (Hrsg.), Perception and Cognition of Music, East Sussex, S. 143-159.

Behne, Klaus-Ernst (2001): Musik-Erleben: Abnutzung durch Überangebot?, in: Media Perspektiven $2001 / 3$, S. 142-147.

Bourdieu, Pierre (1970): Zur Soziologie der symbolischen Formen, Frankfurt / Main.

Bourdieu, Pierre (1982): Die feinen Unterschiede. Kritik der gesellschaftlichen Urteilskraft, Frankfurt / Main.

Bourdieu, Pierre (1983): Ökonomisches Kapital, kulturelles Kapital, soziales Kapital, in: Reinhard Kreckel (Hrsg.), Soziale Ungleichheiten, in: Soziale Welt Sonderband 2, Göttingen, S.183-198.

Bourdieu, Pierre (1998): The State Nobility. Elite Schools in the Field of Power, Stanford.

Bourdieu, Pierre / Jean-Claude Passeron (1977): Reproduction in Society, Education and Culture, London.

Bourdieu, Pierre / Jean-Claude Passeron (1979): The Inheritors. French Students and their Relation to Culture, Chicago.

Bourdieu, Pierre / Luc Boltanski / Robert Castel / Dominique Schnapper (1981): Eine illegitime Kunst. Die sozialen Gebrauchsweisen der Photographie, Frankfurt / Main.

De Graaf, Nan Dirk / Paul M. De Graaf / Gerbert Kraaykamp (2000): Parental Cultural Capital and Educational Attainment in the Netherlands. A Refinement of the Cultural Capital Perspective, in: Sociology of Education 73, S. 92-111.

Diekmann, Andreas (1995): Empirische Sozialforschung. Grundlagen, Methoden, Anwendungen, Reinbek / Hamburg.

DiMaggio, Paul (1982 a): Cultural Capital and School Success: The Impact of Status Culture Participation on the Grades of U.S. High School Students, in: American Sociological Review 47, S. 189-201.

DiMaggio, Paul (1982 b): Cultural Entrepreneurship in Nineteenth Century Boston, Part II: the Classification and Framing of American Art, in: Media, Culture and Society 4, S. 303-322.

DiMaggio, Paul / Mohr John (1985): Cultural Capital, Educational Attainment, and Marital Selection, in: American Journal of Sociology 90, S. 1231-1261.

DiMaggio, Paul / Toquir Mukhtar (2004): Arts Participation as Cultural Capital in the United States, 1982 - 2002: Signs of Decline?, in: Poetics 32, S. 169-204.

Dumais, Susan A. (2005): Early Childhood Cultural Capital, Parental Habitus, and Teacher's Perceptions, in: Poetics 34, S. 83-107.

Erickson, Bonnie (1996): Culture, Class and Connections, in: American Journal of Sociology 102, S. 217-251.

Fröhlich, Gerhard (1994): Kapital, Habitus, Feld, Symbol. Grundbegriffe der Kulturtheorie bei Pierre Bourdieu, in: Ingo Mörth / Gerhard Fröhlich (Hrsg.), Das symbolische Kapital der Lebensstile. Zur Kultursoziologie der Moderne nach Pierre Bourdieu, Frankfurt / Main, S. 31-54.

Gartman, David (1991): Culture as Class Symbolization or Mass Reification? A Critique of Bourdieu's Distinction, in: American Journal of Sociology 97, S. 421-447.

Gebesmair, Andreas (2001): Grundzüge einer Soziologie des Musikgeschmacks, Wiesbaden.

Gebesmair, Andreas (2004): Renditen der Grenzüberschreitung. Zur Relevanz der Bourdieuschen Kapitaltheorie für die Analyse sozialer Ungleichheiten, in: Soziale Welt 55, S. 181-204.

Halle, David (1984): America's Working Man: Work, Home, and Politics Among Blue-Collar Property Owners, Chicago.

Halle, David (1993): Inside Culture: Art and Class in the American Home, Chicago. 
Hartmann, Peter H. (1999): Lebensstilforschung. Darstellung, Kritik und Weiterentwicklung, Opladen.

Holt, Douglas B. (1997): Distinction in America? Recovering Bourdieu's Theory of Tastes from its Critics, in: Poetics 25, S. 93-120.

Holt, Douglas B. (1998): Does Cultural Capital Structure American Consumption?, in: Journal of Consumer Research 25, S. 1-25.

Katsilis, John / Richard Rubinson (1990): Cultural Capital, Student Achievement, and Educational Reproduction: The Case of Greece, in: American Sociological Review 55, S. 270-279.

Kieserling, André, (2008): Felder und Klassen. Pierre Bourdieus Theorie der modernen Gesellschaft, in: Zeitschrift für Soziologie 37, S. 3-24.

Lamont, Michèle, (1992): Money, Morals \& Manners. The Culture of the French and the American UpperMiddle Class, Chicago.

Lareau, Annette / Michèle Lamont (1988): Cultural Capital: Allusions, Gaps and Glissandos in Recent Theoretical Developments, in: Sociological Theory 6, S. 153-168.

Lareau, Annette / Elliot B. Weininger (2003): Cultural Capital in Educational Research: A Critical Assessment, in: Theory and Society 32, S. 567-606.

Lechner, Götz (2002): Ist die Erlebnisgesellschaft in Chemnitz angekommen?, Opladen.

Lehmann, Andreas C. (1993): Habituelle und situative Rezeptionsweisen beim Musikhören im interkulturellen Vergleich, in: Jahrbuch der deutschen Gesellschaft für Musikpsychologie 10, S. 38-55.

Lehmann, Andreas, C. (1994): Habituelle und situative Rezeptionsweisen beim Musikhören. Eine Einstellungstheoretische Untersuchung, Frankfurt / Main.

Levine, Lawrence W. (1990): Highbrow / Lowbrow. The Emergence of Cultural Hierarchy in America, Cambridge.

Müller, Hans-Peter (1986): Kultur, Geschmack und Distinktion. Grundzüge der Kultursoziologie Pierre Bourdieus, in: Friedhelm Neidhardt / Rainer M. Lepsius / Johannes Weiß (Hrsg.), Kultur und Gesellschaft, Opladen, S. 162-190.

Ostrower, Francie (1998): The Arts as Cultural Capital Among Elites: Bourdieu's Theory Reconsidered, in: Poetics 26, S. 43-53.

Otte, Gunnar (2004): Sozialstrukturanalyse mit Lebensstilen. Eine Studie zur theoretischen und methodischen Neuorientierung der Lebensstilforschung, Wiesbaden.

Parcel, Toby / Mikaela Dufur (2001): Capital at Home and at School. Effects on Students Achievement, in: Social Forces 79, S. 881-912.

Peterson, Richard A. / Roger M. Kern (1996): Changing Highbrow Taste: From Snob to Omnivore, in: American Sociological Review 61, S. 900-907.

Reuband, Karl-Heinz (2007): Partizipation an der Hochkultur und die Überschätzung kultureller Kompetenz, in: Österreichische Zeitschrift für Soziologie 32, S. 46-70.

Robinson, Robert V. / Maurice Garnier (1985): Class Reproduction Among Men and Women in France: Reproduction Theory On Its Home Grounds, in: American Journal of Sociology 91, S. 250-280.

Roose, Henk (2008): Many-Voiced or Unisono? An Inquiry into Motives for Attendance and Aesthetic Dispositions of the Audience Attending Classical Concerts, in: Acta Sociologica 51, S. 237-253.

Rössel, Jörg (2004): Von Lebensstilen zu kulturellen Präferenzen - Ein Vorschlag zur theoretischen Neuorientierung, in: Soziale Welt 55, S. 95-114.

Rössel, Jörg (2005): Plurale Sozialstrukturanalyse. Eine Rekonstruktion der Grundbegriffe der Sozialstrukturanalyse, Wiesbaden.

Rössel, Jörg / Claudia Beckert-Zieglschmid (2002): Die Reproduktion kulturellen Kapitals, in: Zeitschrift für Soziologie 31, S. 497-519.

Rössel, Jörg / Rolf Hackenbroch / Angela Göllnitz (2002): Die soziale und kulturelle Differenzierung des Hochkulturpublikums, in: Sociologia Internationalis 40, S. 191-212. 
Rössel, Jörg; / Angela Göllnitz / Rolf Hackenbroch (2005): Soziale Differenzierung und Strukturwandel des Hochkulturpublikums, in: Institut für Kulturpolitik der Kulturpolitischen Gesellschaft (Hrsg.): Jahrbuch für Kulturpolitik 200, Essen, S. 225-234.

Savage, Mike / James Barlow (1992): Property, Bureaucracy and Culture, London.

Schulze, Gerhard (1992): Die Erlebnisgesellschaft. Frankfurt / Main.

Sullivan, Alice (2001): Cultural Capital and Educational Attainment, in: Sociology 35, S. 893- 912.

van Eijck, Koen (2001): Social Differentiation in Musical Taste Patterns, in: Social Forces 79, S. 1163-1184.

van Eijck, Koen / Bertine Bargeman (2004): The Changing Impact of Social Background on Lifestyle: Culturalization Instead of Individualization?, in: Poetics 32, S. 439-461.

Prof. Dr. Jörg Rössel Soziologisches Institut Universität Zürich Andreasstrasse 15 CH-8050 Zürich roessel@soziologie.uzh.ch 


\section{Anhang}

Anhang Tabelle 1: Items zur Erfassung der Musikrezeption

\begin{tabular}{|c|c|c|}
\hline Itemformulierung & $\begin{array}{c}\text { Mittel- } \\
\text { wert }\end{array}$ & Standardabweichung \\
\hline ... summe ich gerne mit. & 2,07 & 1,24 \\
\hline $\begin{array}{l}\text {... soll sie mich auf andere Gedanken bringen, unangenehme Stim- } \\
\text { mungen aus meinem Kopf vertreiben. }\end{array}$ & 3,33 & 1,23 \\
\hline $\begin{array}{l}\text {... versuche ich gleich zu erkennen, welche Art von Oper das sein } \\
\text { könnte. }\end{array}$ & 2,77 & 1,38 \\
\hline ... möchte ich mich am liebsten immer bewegen. & 2,05 & 1,08 \\
\hline $\begin{array}{l}\text {...finde ich es interessant, die verschiedenen Themen, Melodien } \\
\text { und Rhythmen zu verfolgen. }\end{array}$ & 3,86 & 1,01 \\
\hline ... „bade“ ich gerne in den Klängen der Musik. & 3,96 & 1,07 \\
\hline ... kann es sein, dass mir die Musik regelrecht unter die Haut geht. & 4,11 & 0,87 \\
\hline $\begin{array}{l}\text {... höre ich gern nur mit einem } \mathrm{Ohr} z u \text { und denke dabei an etwas } \\
\text { anderes. }\end{array}$ & 2,03 & 0,99 \\
\hline ... versuche ich den Formaufbau des Stückes zu verstehen. & 2,88 & 1,27 \\
\hline ... höre ich vor allem mit dem Gefühl. & 3,88 & 0,97 \\
\hline $\begin{array}{l}\text {... kann es sein, dass ich bestimmte körperliche Wirkungen (Herz- } \\
\text { schlag, Kribbeln, Gefühl im Magen) spüre. }\end{array}$ & 3,15 & 1,24 \\
\hline $\begin{array}{l}\ldots \text { achte ich darauf, wie der Komponist die Melodien, den Rhyth- } \\
\text { mus oder die Harmonien gestaltet hat. }\end{array}$ & 2,99 & 1,23 \\
\hline $\begin{array}{l}\text {... kann es sein, dass ich meine Gefühle und Stimmungen in der } \\
\text { Musik wiederfinde. }\end{array}$ & 3,57 & 0,99 \\
\hline $\begin{array}{l}\text {... habe ich oft irgendwelche Gedanken, die mit der Musik selbst } \\
\text { gar nichts zu tun haben. }\end{array}$ & 2,42 & 1,03 \\
\hline ... kann es sein, dass ich am liebsten weinen möchte. & 2,30 & 1,12 \\
\hline ... kann es sein, dass der Rhythmus mich ganz gefangen hält. & 3,43 & 0,95 \\
\hline ... fühle ich mich weniger einsam. & 2,45 & 1,25 \\
\hline $\begin{array}{l}\text {... achte ich darauf, ob sich z. B. die Tonart oder etwas anderes } \\
\text { ändert. }\end{array}$ & 2,26 & 1,21 \\
\hline ... träume ich am liebsten. & 2,73 & 1,13 \\
\hline $\begin{array}{l}\text {... achte ich darauf, welche Gefühe durch die Musik ausgedrückt } \\
\text { werden. }\end{array}$ & 3,54 & 0,99 \\
\hline $\begin{array}{l}\text {... achte ich darauf, ob die Musiker das Stück auch wirklich gut } \\
\text { spielen. }\end{array}$ & 3,39 & 1,24 \\
\hline $\begin{array}{l}\text {... finde ich es gut, wenn man sich der Musik ganz hingeben kann, } \\
\text { sich ganz in sie verstecken kann. }\end{array}$ & 3,55 & 1,09 \\
\hline ... steht die Musik des Orchesters im Vordergrund. & 3,18 & 0,97 \\
\hline ... steht der Gesang im Vordergrund. & 3,60 & 1,01 \\
\hline
\end{tabular}

Die Eingangsformulierung lautet: Wenn ich Musik in der Oper höre, ... Die Befragten hatten eine fünfstufige Antwortskala zur Verfügung, die von „nie“ (1) bis „fast immer“ (5) reichte. 
Anhang Tabelle 2: Musikkulturelle Partizipation

\begin{tabular}{lcc}
\hline & „Mittlerer“ Geschmack & Hochkultur \\
\hline Operette & 0,861 & --- \\
Musical & 0,860 & -- \\
Oper & 0,378 & 0,707 \\
Ballett & --- & 0,658 \\
Konzerthaus & -- & 0,510 \\
Erklärte Varianz in \% & 38,301 & 24,896 \\
\hline
\end{tabular}

Faktorladungen kleiner als 0,3 werden nicht angezeigt.

Anhang Tabelle 3: Musikalische Bildung

\begin{tabular}{lcc}
\hline & Aktives Musizieren & Kulturelles Klima im Elternhaus \\
\hline Instrument gelernt & 0,794 & --- \\
Musikschule besucht & 0,796 & --- \\
Musiziere noch heute & 0,796 & --- \\
Eltern musikinteressiert & --- & 0,782 \\
Schon als Kind in Oper & --- & 0,836 \\
Erklärte Varianz in \% & 43,083 & 22,846 \\
\hline
\end{tabular}

Faktorladungen kleiner als 0,3 werden nicht angezeigt. 